\title{
An anatomic study of the accessory anterolateral talar facet
}

\author{
K. Hattori' ${ }^{1}$, E. Sakuma ${ }^{2}$, M. Nakayama ${ }^{2}$, A. Kozaki², I. Wada ${ }^{1}$, T. Otsuka ${ }^{1}$ \\ ${ }^{1}$ Department of Orthopaedic Surgery, Nagoya City University Graduate School of Medical Sciences, Nagoya, Japan \\ 2Department of Functional Anatomy, Nagoya City University Graduate School of Medical Sciences, Nagoya, Japan
}

[Received 10 June 2014; Accepted 22 July 2014]

Background: A small accessory facet with articular surface morphology is occasionally seen on the talus, bordering on the lateral end of the sinus tarsi. This facet has been named the accessory anterolateral talar facet. However, few anatomical studies have addressed this facet. Here we present the precise morphology of accessory anterolateral talar facet with emphasis on anatomical correlation between the presence of this facet and the angle of the infero-lateral surface of the talus (talar infero-lateral surface - TILS angle).

Materials and methods: A total of 22 (11 male, 11 female) adult cadavers with no known pathological conditions in the talocalcaneal joints were examined during educational dissection at Nagoya City University Medical School in 2013. After exclusion of 1 joint due to the poor condition of the talus, 43 talus (22 right, 21 left) were analysed. We judged the presence of the accessory anterolateral talar facet and measured TILS angle. We performed statistical analysis on the point of laterality, gender difference and the difference in the TILS angles in tali with or without the accessory anterolateral talar facets.

Results: An accessory anterolateral talar facet was identified in 11 (26\%) of the 43 specimens. Of the 21 cadavers with paired talar specimens, 5 displayed the facet bilaterally.

Conclusions: There was no sex difference and no significant laterality, however we found that TILS angle was significantly larger in accessory anterolateral talar facet positive samples than in negative ones. (Folia Morphol 2015; 74, 1: 61-64)

Key words: talus, anatomy, cadaver, variation, facet

\section{INTRODUCTION}

Many patients with symptomatic flatfoot deformity often present some pain in the lateral part of the hindfoot. A well-known reason for this lateral pain is talocalcaneal impingement. A small accessory facet with articular surface morphology is occasionally seen on the talus, bordering on the lateral end of the sinus tarsi which is a main location where symptoms of talocalcaneal impingement occur. This facet has been named the accessory anterolateral talar facet. Recently, there have been some reports that the accessory anterolateral talar facet might represent a new aetiology for the lateral pain in the rigid flatfoot. Martus et al. [7] presented a retrospective review of patients with painful talocalcaneal impingement associated with an accessory anterolateral talar facet treated operatively. In their case series, facet resection provided good results with early pain relief and return to normal function.

However, there have been few anatomical investigations concerning the relation between the presence 
of the accessory anterolateral talar facet and the variation of measured angles within the talocalcaneal joints. This lack hampers clinical decision making in patients with related symptoms of painful talocalcaneal impingement in the rigid flatfoot. To understand the reason for the pain originating from the accessory anterolateral talar facet its precise morphology needs to be studied.

In the present study, we report the morphometry for the accessory anterolateral talar facet in feet from the fixed cadavers of elderly people with emphasis on anatomical correlation between the presence of this facet and the angle of the infero-lateral surface of the talus (talar infero-lateral surface - TILS angle).

\section{MATERIALS AND METHODS}

The present study was conducted within the parameters of the written permissions we received from the donors and their surviving relatives and the present work conformed to the provisions of the Declaration of Helsinki in 1995 (as revised in Edinburgh in 2000). This study was approved by the institutional review board of Nagoya City University Graduate School of Medical Sciences according to the ethical guidelines for epidemiology research (Approved Number 966).

A total of 22 (11 male, 11 female) adult cadavers with no known pathological conditions in the talocalcaneal joints were examined during educational dissection at Nagoya City University Medical School in 2013. The average age at death was 78.9 (64-90) years in males and $92.5(82-105)$ years in females. Because of the poor condition of the talus, analysable data could not be obtained from 1 case. After exclusion of this 1 case, 43 talus (22 right, 21 left) were analysed (Tables 1, 2). All of the cadavers had been perfused by using fixative through the femoral artery and preserved in a natural posture in the usual manner. We transected and reflected the skin, ligaments and soft tissues to study the presence or absence of an accessory anterolateral talar facet. After detailed dissections of the lateral surface of each talus to make it completely visible, the morphometric measurements were taken.

The maximum anteroposterior width of the accessory anterolateral talar facet was measured using a Vernier calliper (KSM-15FF, Nakamura Mfg. Co., Ltd, Tokyo, Japan). We judged the talus as positive when this measurement was equal to or exceeded $5 \mathrm{~mm}$.
Table 1. Male

\begin{tabular}{ccccc}
\hline & Right & ALTF & Left & ALTF \\
\hline 1 & $82^{\circ}$ & - & $80^{\circ}$ & - \\
2 & $90^{\circ}$ & - & $88^{\circ}$ & - \\
3 & $84^{\circ}$ & - & $89^{\circ}$ & - \\
4 & $110^{\circ}$ & + & $119^{\circ}$ & + \\
5 & $115^{\circ}$ & + & $100^{\circ}$ & + \\
6 & $87^{\circ}$ & - & $87^{\circ}$ & - \\
7 & $107^{\circ}$ & + & $114^{\circ}$ & + \\
8 & $89^{\circ}$ & - & $90^{\circ}$ & - \\
9 & $72^{\circ}$ & - & $74^{\circ}$ & - \\
10 & $84^{\circ}$ & - & $86^{\circ}$ & - \\
11 & $90^{\circ}$ & - & $83^{\circ}$ & - \\
A & $91.8^{\circ}$ & & $91.8^{\circ}$ & \\
\hline
\end{tabular}

The presence of accessory anterolateral talar facet (ALTF) and talar infero-lateral surface angle; $\mathrm{A}$ - average

Table 2. Female

\begin{tabular}{ccccc}
\hline & Right & ALTF & Left & ALTF \\
\hline 1 & $108^{\circ}$ & + & $107^{\circ}$ & + \\
2 & $69^{\circ}$ & - & $68^{\circ}$ & - \\
3 & $92^{\circ}$ & - & $90^{\circ}$ & - \\
4 & $88^{\circ}$ & - & $94^{\circ}$ & - \\
5 & $89^{\circ}$ & - & $92^{\circ}$ & - \\
6 & $112^{\circ}$ & + & $110^{\circ}$ & + \\
7 & $108^{\circ}$ & + & $92^{\circ}$ & - \\
8 & $102^{\circ}$ & - & $113^{\circ}$ & - \\
9 & $73^{\circ}$ & - & $87^{\circ}$ & - \\
10 & $104^{\circ}$ & - & $N A$ & NA \\
11 & $79^{\circ}$ & - & $78^{\circ}$ & - \\
A & $93.1^{\circ}$ & & $93.1^{\circ}$ & \\
\hline
\end{tabular}

ALTF — anterolateral talar facet; NA — not available due to the poor condition of the talus; $\mathrm{A}$ - average

We also measured the angle defined by the infero-lateral surfaces of the lateral process of the talus (TILS angle) by using an orthopaedic protractor (Fig. 1).

Statistical analyses on the point of laterality, gender difference and the difference in the TILS angles in tali with or without the accessory anterolateral talar facets were obtained by using Student's t-test method.

\section{RESULTS}

We found the accessory anterolateral talar facet being situated at the junction of the lateral surface of the corpus with the posterior wall of the sinus tarsi, in front and somewhat to the outer side of the lateral 


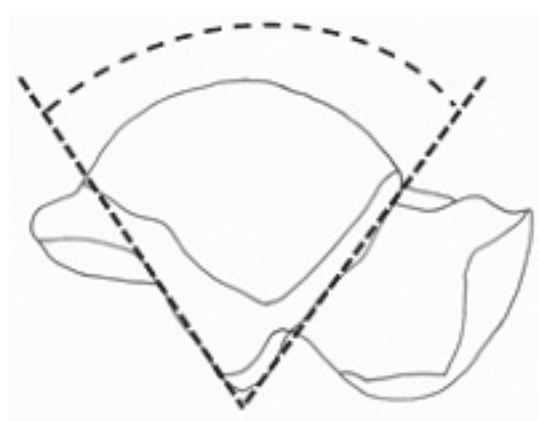

Talar infero-lateral surface angle*

Fiigure 1. Lateral view of talus; *the angle defined by the infero-lateral surfaces of the lateral process of the talus.

Table 3. Sex difference of talar infero-lateral surface angle

\begin{tabular}{lcc}
\hline & Male & Female \\
\hline Mean \pm SD & $91.8 \pm 13.2$ & $93.1 \pm 14.2$ \\
\hline
\end{tabular}

No significant difference $(p>0.05)$; SD - standard deviation

Table 4. Laterality of talar infero-lateral surface angle

\begin{tabular}{ccc}
\hline & Right & Left \\
\hline Mean \pm SD & $92.4 \pm 13.6$ & $92.5 \pm 13.8$ \\
\hline
\end{tabular}

No significant laterality $(p>0.05)$; SD — standard deviation

Table 5. Talar infero-lateral surface (TILS) angle with or without accessory anterolateral talar facet (ALTF)

\begin{tabular}{ccc}
\hline & $\begin{array}{c}\text { ALTF } \\
\text { positive }\end{array}$ & $\begin{array}{c}\text { ALTF } \\
\text { negative }\end{array}$ \\
\hline Mean \pm SD & $110.0 \pm 5.0$ & $86.4 \pm 9.6$ \\
\hline
\end{tabular}

TILS angle of positive group is larger than that of negative group $(p<0.05)$; $\mathrm{SD}$ - standard deviation

process of the talus. Articular cartilage was always observed on the accessory facet and the cartilage surface faced forwards and slightly outwards. The accessory facet could be identified when there was an anteriorly directed prominence of the lateral talar process with a surface appearance similar in character to other talar articulations. The accessory facet appeared somewhat triangular in shape and was easily distinguished from the regular talus.

In this investigation, an accessory anterolateral talar facet was identified in 11 (26\%) of the 43 specimens. Of the 21 cadavers with paired talar specimens, 5 displayed the facet bilaterally.
We found no significant difference in the TILS angle between the male and female donors (Table 3). Also, there was no significant difference in the angles measured in right and left legs (Table 4).

The TILS angle was significantly larger in accessory anterolateral talar facet positive samples than in negative ones (Table 5).

\section{DISCUSSION}

The acquired deformity of peroneal spastic flatfoot was first described by Robert Jones in 1897 [3]. It is known that the painful flatfoot may occur after puberty and in early adolescence. The "spastic" flatfoot does not require a constant state of peroneal contraction, although active spasm may be produced by irritation of the painful hindfoot with motion and weight bearing. Chronic subtalar eversion may result in adaptive shortening of the peroneal musculature [2].

While impingement between the talus and calcaneus has been suggested as a cause of pain in the lateral part of the hindfoot, the precise origin of the pain has not been clarified. The accessory anterolateral talar facet is situated at the lateral rim of the sinus tarsi. It is known that the sinus tarsi opens in inversion and closes in eversion $[5,9]$. Thus, hindfoot valgus leads to a narrowing of the sinus tarsi due to the eversion. Moreover, it is reasonable that eversion beyond the normal range might cause abnormal osseous contact in the lateral side of the sinus tarsi, because subluxation of the talocalcaneal joint commonly occurs in this population [1].

Malicky et al. [6] examined the computed tomography (CT) of 19 adult patients with symptomatic flatfoot, with use of a custom loading frame designed to simulate weight-bearing. The frame was designed to hold the foot in a neutral position with axial compressive load applied to determine the potential causes of pain in the lateral aspect of the foot. They described that lateral hindfoot impingement occurred within the sinus tarsi [6].

The role played by the accessory anterolateral talar facet in symptomatic sinus tarsi impingement could make it an important therapeutic target.

Among previous studies on the talocalcaneal articulation, Kelikian and Sarrafian [4] found that a large accessory surface was present in $4 \%$, and an accessory surface of variable size was present in $34 \%$ in a series of 100 tali of cadaver specimens.

The accessory anterolateral talar facet was first described by Sewell [10] in 1904. Sewell made a study in a series of Egyptian bones amounting to 1,006 talar specimens. He characterised numerous 
anatomical variants of the talus; including the accessory anterolateral talar facet which he termed "facies externa accessorcia corporis tali". He noted that this facet was found to occur in 102 cases in the 1,006 bones being examined, thus giving $10.15 \%$ [10].

Martus et al. [7, 8] surveyed the paediatric bone samples within the Hamann-Todd Human Osteological Collection at the Cleveland Museum of Natural History, and studied the 79 paired tali and calcanei from the 43 skeletons from individuals who had an average age of 13.4 years (range 3-18 years) at the time of death. In their investigations, an accessory anterolateral talar facet was identified in $27(34 \%)$ of the 79 specimens and was large in $2(2.5 \%)$.

In our study, we counted only those facets with a sagittal plane width of $5 \mathrm{~mm}$ or more as positive, while the measurement technique was only briefly described in the previous reports. Therefore, the disparities among the studies including the present study concerning the occurrence of the accessory anterolateral talar facet might reflect the methodological differences and definition rules.

Martus et al. [7, 8] also reported that the accessory anterolateral talar facet was significantly more frequent among specimens from male donors $(63 \% ; 12$ of 19$)$ than female donors ( $21 \% ; 5$ of 24$)$. A possible explanation for the difference between these results and ours concerning gender could be the difference of average age between male and female specimens. In our study, the average age at the death in female (92.5 years) was higher than that of male (78.9 years). Sewell [10] has suggested that the accessory anterolateral facets show progressive hypertrophy. Supposing that this is correct, the accessory anterolateral talar facets of the female samples in our series may have hypertrophied more with aging compared with those of the male samples.

Statistical analyses in the present study showed that the tali with an accessory anterolateral talar facet had a significantly larger TILS angle in comparison with those without the facet. Martus et al. $[7,8]$ have made the conclusion that both the presence and the forward prolongation of this facet were to be attributed to the influence of weight bearing. We also surmise that the accessory anterolateral talar facets hypertrophy in an anterior direction, leading to larger TILS angles than those of facet negative samples.

We also believed that this angle difference might be a causative factor in the onset of inflammation in the soft tissues such as talocalcaneal ligaments in people with painful flatfoot who have the accessory anterolateral talar facet.

\section{CONCLUSIONS}

Non-invasive imaging techniques, such as CT and magnetic resonance imaging (MRI), have made it possible to demonstrate anatomical abnormalities which have been difficult to detect. In the orthopaedic assessment of the lateral part of the sinus tarsi including the accessory anterolateral talar facet, cross-sectional imaging such as $\mathrm{CT}$ with coronal and sagittal reconstructions might be useful for morphological identification and MRI could be a great help in demonstrating the evidences of impingement through localised oedema of bone marrow. The possibility of an accessory anterolateral talar facet should always be kept in mind during the clinical diagnoses of lateral foot pain in talocalcaneal impingement presenting with rigid flatfoot.

\section{REFERENCES}

1. Ananthakrisnan D, Ching R, Tencer A, Hansen ST Jr, Sangeorzan BJ (1999) Subluxation of the talocalcaneal joint in adults who have symptomatic flatfoot. J Bone Joint Surg Am, 81: 1147-1154.

2. Blockey NJ (1955) Peroneal spastic flat foot. J Bone Joint Surg Br, 37: 191-202.

3. Jones R (1897) Peroneal spasm, and its treatment. Liverpool Med Chir J, 17: 442.

4. Kelikian AS, Sarrafian SK ed. Sarrafian's anatomy of the foot and ankle (2011) Descriptive, topographic, functional. 3rd Ed. Lippincott Williams \& Wilkins, Philadelphia, pp. 48-61.

5. Knudson GA, Kitaoka HB, Lu CL, Luo ZP, An KN (1997) Subtalar joint stability. Talocalcaneal interosseous ligament function studied in cadaver specimens. Acta Orthop Scand, 68: 442-446.

6. Malicky ES, Crary JL, Houghton MJ, Agel J, Hansen ST Jr, Sangeorzan BJ (2002) Talocalcaneal and subfibular impingement in symptomatic flatfoot in adults. J Bone Joint Surg Am, 84: 2005-2009.

7. Martus JE, Femino JE, Caird MS, Kuhns LR, Craig CL, Farley FA (2008) Accessory anterolateral talar facet as an etiology of painful talocalcaneal impingement in the rigid flatfoot: a new diagnosis. lowa Orthop J, 28:1-8.

8. Martus JE, Femino JE, Caird MS, Hughes RE, Browne RH, Farley FA (2008) Accessory anterolateral facet of the pediatric talus. An anatomic study. J Bone Joint Surg Am, 90: 2452-2459.

9. Ozeki S, Kitaoka H, Uchiyama E, Luo ZP, Kaufman K, An KN (2006) Ankle ligament tensile forces at the end points of passive circumferential rotating motion of the ankle and subtalar joint complex. Foot Ankle Int, 27: 965-969.

10. Sewell R (1904) A study of the astralagus. Part II. J Anat Physiol, 38: 423-432. 